\title{
Registro fotográfico de tres especies de felinos (Carnivora: Felidae) simpátricos en el Parque Nacional Machalilla, costa del Ecuador
}

\author{
Joselyn Geomayra Belduma Garcia ${ }^{1 *}$ iD , Edwin Leandro Bailón Burgos ${ }^{1}$ (D), Katheryn \\ Ariel Rodríguez De Mera1, Angie Estefanía Carreño Zambrano', Luis Bryan Chérrez \\ Palma1 ${ }^{\text {iD }}$, Andrés Hernán Espinoza Espinal ${ }^{1}$ (D), Miguel Ángel Méndez Chalar ${ }^{1}$, Enri- \\ que de la Montaña1id
}

1 Facultad de Ciencias Agropecuarias, Universidad Laica Eloy Alfaro de Manabí, Ciudadela Universitaria, Vía a San Mateo, 130802, Manta, Ecuador

* Correspondencia: geomayra2550.gb@gmail.com

\section{Resumen}

El Parque Nacional Machalilla (PNM) ubicado en el hotspot Tumbes-Chocó-Magdalena al oeste de Ecuador, contiene altos niveles de biodiversidad y endemismo; sin embargo, presenta una constante pérdida y degradación de su hábitat debido a la actividad antrópica. En este trabajo se determinó la presencia de los felinos silvestres dentro del área protegida del PNM. Se ubicaron 60 cámaras trampa dentro de las 56. 184 ha de la zona terrestre del PNM, entre los meses de octubre 2018 y febrero de 2019. Durante este periodo se detectó la presencia de tres especies de felinos silvestres: ocelote (Leopardus pardalis), margay (Leopardus wiedii) y yaguarundí (Herpailurus yagouaroundi). En esta nota se presenta un caso en particular en el que se reportó la presencia de las tres especies de felinos coincidiendo en una de las cámaras trampa.

Palabras clave: hotspot, cámaras trampa, Manabí, solapamiento de hábitat.

\section{Abstract}

The Machalilla National Park (PNM) is located in the Tumbes-Chocó-Magdalena hotspot in western Ecuador, and contains high levels of biodiversity and endemism; however, it presents a constant loss and degradation of its habitat due to anthropogenic activity. In this work, the presence of wild cats within the protected area of the PNM was determined. 60 trap cameras were located within the 56,184 ha of the PNM land area, between the months of October 2018 and February 2019. During this period, the presence of three species of wild cats was detected: ocelot (Leopardus pardalis), margay (Leopardus wiedii) and yaguarundi (Herpailurus yagouaroundi). This note presents a particular case in which the presence of the three feline species was reported coinciding in one of the trap chambers.

Key words: hotspot, camera trap, Manabí, habitat overlap. 
Las presiones humanas han generado un aislamiento paulatino de las poblaciones de felinos y la disminución de individuos en su hábitat natural (Clavijo et al. 2009). En el Neotrópico las especies de la familia Felidae son de las más afectadas por las actividades humanas (Ceballos et al. 2010). De las 37 especies de felinos existentes en el mundo, siete se ubican en Ecuador (Tirira, 2019), siendo vulnerables ante la pérdida y transformación del hábitat, disminución de presas, comercio ilegal de sus pieles y cacería por retaliación (Payán y Soto, 2012). En el único trabajo realizado en este siglo en el área de estudio se han registrado tres especies de felinos (Cervera et al. 2016): la especie margay (Leopardus wiedii), categorizado como "Casi Amenazado" por la Unión Internacional para la Conservación de la Naturaleza (UICN), y el ocelote (Leopardus pardalis) y yaguarundí (Herpailurus yagouaroundi) categorizados como "Preocupación Menor".

A pesar de la importancia ecológica de estas especies, la información sobre la dinámica ecológica de los felinos en la región Costa de Ecuador es escasa ya que la mayoría de los estudios son enfocados en la caracterización de la flora y fauna en zonas protegidas de la región Amazónica del país (Ortega-Andrade et al. 2015). Los resultados de este trabajo forman parte de un proyecto de monitoreo de los mamíferos medianos y grandes presentes en el Parque Nacional Machalilla (PNM); (lat: -1,54000, lon: -80,740000), en plena región tumbesina del litoral ecuatoriano (Figura 1).

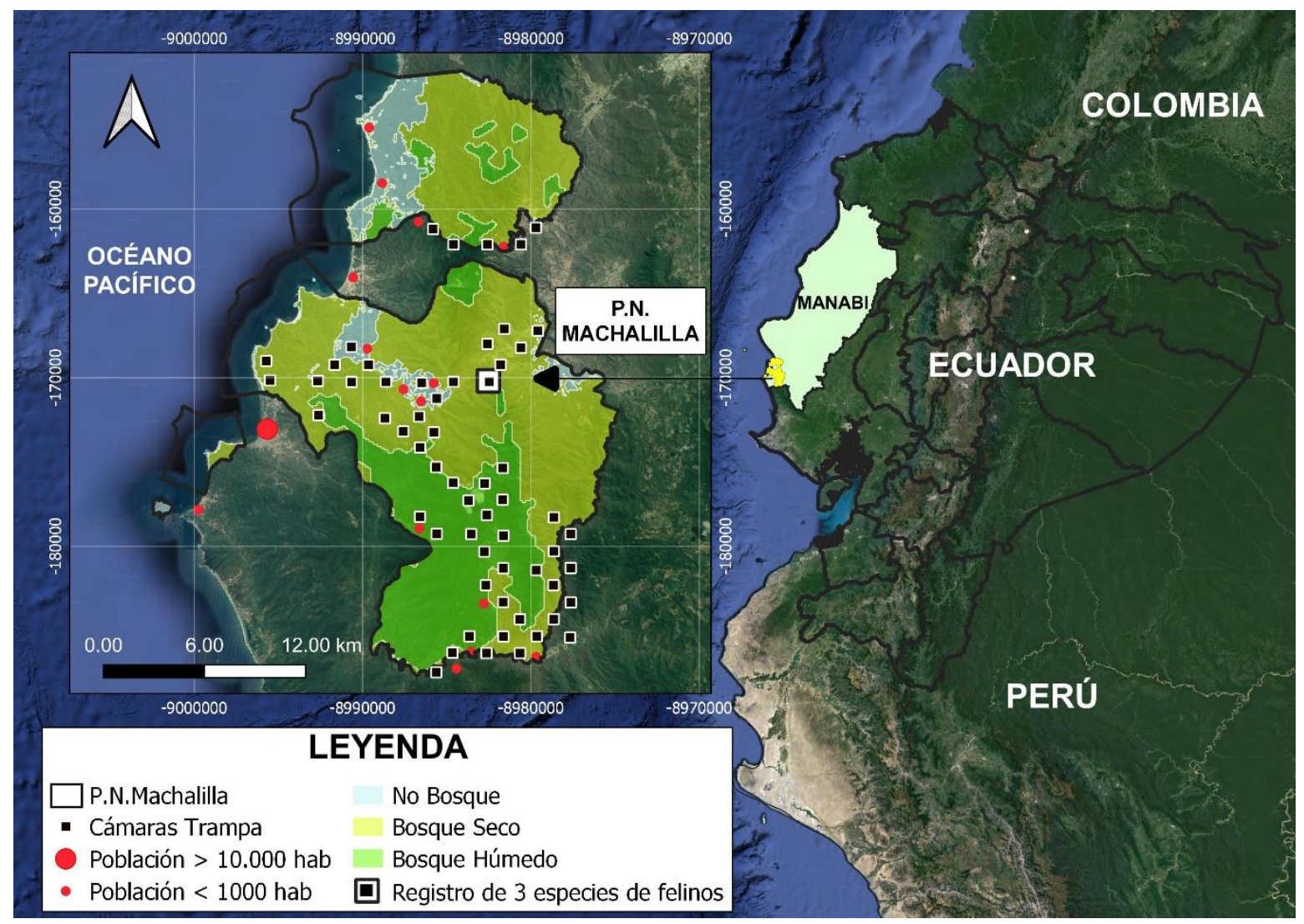

FIGURA 1. Área de estudio con la ubicación de las 60 estaciones de fototrampeo instaladas en el Parque Nacional Machalilla, provincia de Manabí, Ecuador.

En esta nota solo se presentan los registros obtenidos en una de las cámaras trampa, en la que se detectó la presencia de tres especies de felinos. El PNM es el único parque nacional de la región Costa y el principal enclave para la conservación del bosque tropical 
estacional seco; la temperatura media anual es de $24^{\circ} \mathrm{C}$, con una precipitación media anual de 758 mm y una marcada estacionalidad; una temporada seca (junio - noviembre) con 147 $\mathrm{mm}$ y una temporada húmeda (diciembre - mayo) con $613 \mathrm{~mm}$ de precipitación. Comprende dos zonas, una terrestre con 56.184 ha y una marina con 14.430 ha. Sin embargo, estos ecosistemas se enfrentan a elevados niveles de fragmentación de hábitat, con tasas de deforestación anual promedio de 94. 353 ha/año (MAE, 2017).

Se ubicaron 60 cámaras trampa en la zona continental del PNM, entre los meses de octubre 2018 y febrero 2019, durante la temporada seca de la región. Las cámaras trampas digitales fueron de la marca Moultrie M990i (PRADCO, Birmingham, AL, USA), ubicadas con $1 \mathrm{~km}$ de separación, sujetas a un árbol a una altura de 30-50 cm sobre el nivel del suelo y programadas para tomar ráfagas de tres fotografias con un intervalo de cinco segundos, permaneciendo activas las 24 horas durante un periodo de 45 días aproximadamente (TEAM, 2011).

Durante la actividad de fototrampeo se obtuvo un esfuerzo de muestreo de 1.847 díascámaras, con un total de 56.873 fotografias de vertebrados terrestres; de las cuales 376 pertenecen a la familia Felidae presentes en 21 sitios de muestreo. Entre las especies de felinos $L$. pardalis fue registrado en 16 sitios (312 fotografias), mientras que L. wiedii observado en siete sitios (47 fotografias) y H. yagouaroundi en dos sitios (17 fotografias). En ningún caso se pudo constatar la presencia de hembras preñadas o con crías.

En una de las estaciones de muestreo se observó particularmente la presencia de las tres especies de felinos antes mencionadas (lat: -1,528997145, lon: -80,69099974; 333 m). La zona donde se obtuvieron los registros es la característica de los remanentes de bosque semideciduo de tierras bajas del centro del PNM (Figura 2). Estos bosques se localizan en lugares con pendientes fuertes y un estrato arbóreo disperso, formado por árboles que alcanzan los $15 \mathrm{~m}$ de alto, predominando las especies Ceiba trichistandra y Tabebuia chrysantha, además se encuentran gran cantidad de epifitas no vasculares y vasculares. En este sitio se registraron otras dos especies de mesocarnivoros: Eira barbara y Nassua narica; y cinco especies presas de mamíferos: Tamandua mexicana, Simosciurus stramineus, Didelphis marsupialis, Sylvilagus brasiliensis y Odocoileus virginianus. Además, hubo registros de cazadores en el sitio que principalmente acuden en busca de venados de cola blanca (O. virginianus). Como diferencia respecto a otros lugares de muestreo cercanos se puede destacar que en esta estación de muestreo se encontraba una letrina de L. pardalis.

Durante el periodo de muestreo se obtuvieron 30 eventos fotográficos de L. pardalis a partir del 15 de diciembre del 2018. El 80\% de los registros de esta especie fue entre las 19:00 y las 06:00 h, mientras que el 20\% restante de los registros ocurrieron entre las 06:00 y 10:00 h; por lo que esta especie tuvo una actividad principalmente nocturna. En el caso de L. wiedii se obtuvieron seis eventos fotográficos a partir del 29 de diciembre del 2018, y todos ellos fueron nocturnos entre las 20:00 y 06:00 h. Por último, se obtuvieron cuatro eventos fotográficos de la especie H. yagouaroundi, todos ellos diurnos entre las 09:00h y las 17:00h a partir del 11 de febrero del 2019.

En los registros obtenidos de las tres especies se evidenció un solapamiento de hábitat, aunque con uso temporal diferencial. Las especies L. pardalis y L. wiedii registraron mayor actividad nocturna. Los hábitos estrictamente nocturnos de L. wiedii coinciden con estudios realizados en Argentina y México (Di Bitetti et al. 2010; Briones-Salas et al. 2016). 

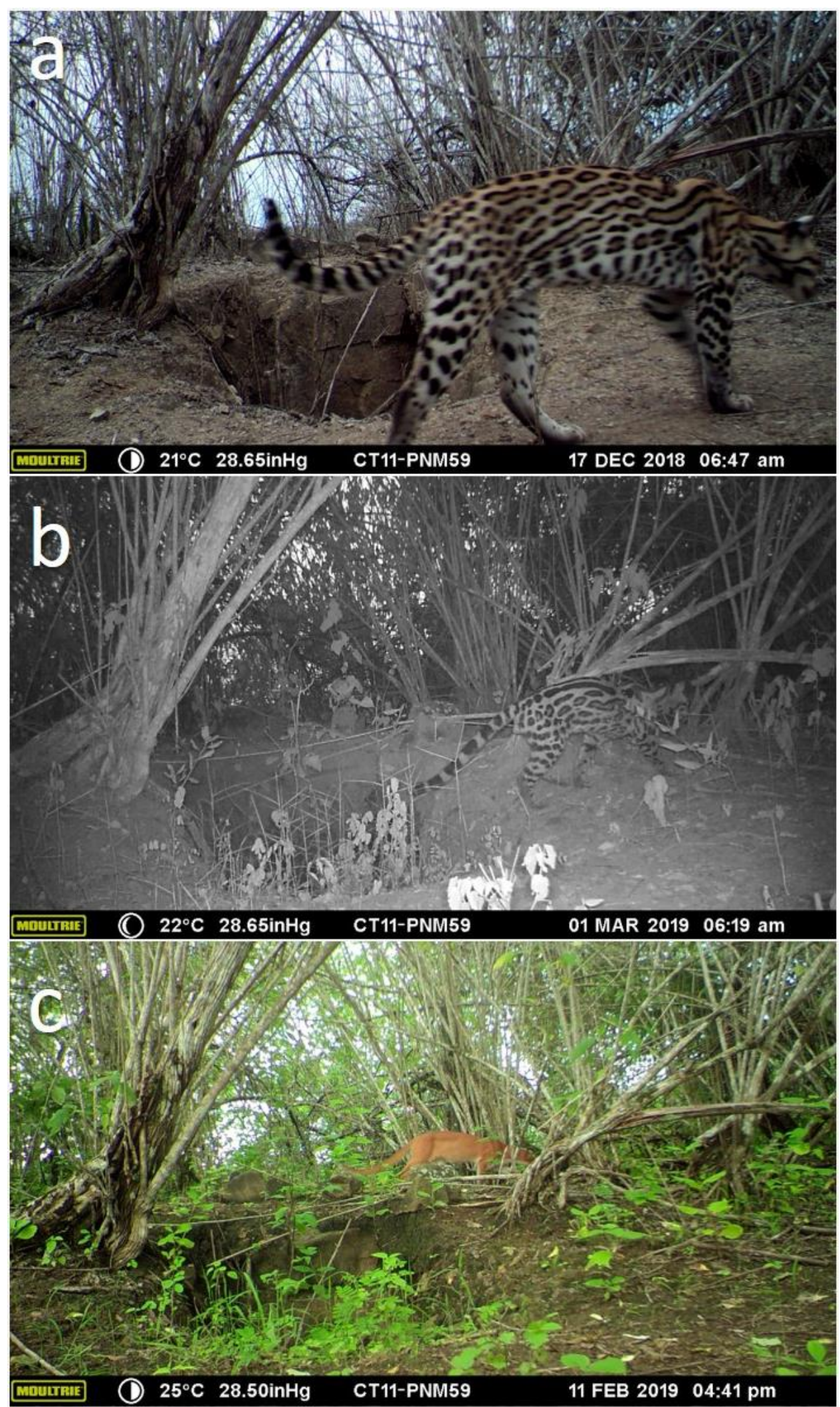

FIGURA 2. Fotografias de especies de felinos silvestres registradas en el sitio de muestreo del Parque Nacional Machalilla a. ocelote (L. pardalis), b. margay (L. wiedii), c. yaguarundí (H. yagouaroundi). 
Por otro lado, L. pardalis presentó un 20\% de actividad diurna, coincidiendo con los resultados del estudio realizado en el norte de Perú, en donde también se registró una pequeña actividad diurna (Kolowski y Alonso, 2010). En cuanto a H. yagouaroundi presentó una actividad estrictamente diurna, coincidiendo con lo reportado previamente en otras regiones (Di Bitetti et al. 2010; Briones-Salas et al. 2016). Debido a que H. yagouaroundi y L. wiedii son felinos que poseen gran similitud morfológica (Kiltie, 1984), el patrón opuesto de actividad mostrado por sus registros probablemente sea el resultado de adaptaciones para reducir la competencia interespecífica por los recursos tróficos (Di Bitetti et al. 2010; Pérez-Irineo y Santos-Moreno, 2014). Por otro lado, L. wiedii presentó un alto solapamiento de sus patrones de actividad con los de L. pardalis, por lo que probablemente segregue el uso del espacio, a través del uso del estrato arbóreo en sitios con presencia de L. pardalis, como sugieren Di Bitetti et al. (2010).

Los resultados de este trabajo muestran que las especies ocelote ( $L$. pardalis), margay ( $L$. wiedii) y yaguarundi ( $H$. yagouaroundi) comparten el mismo hábitat en el PNM, y sugieren la importancia que tiene la segregación de la actividad temporal como un mediador de las interacciones interespecíficas en estas especies (Carrera-Treviño et al. 2018). Con los datos disponibles no podemos conocer las causas que subyacen detrás de que estas tres especies simpátricas sean registradas raras veces compartiendo un mismo lugar del PNM, por lo que consideramos que son necesarias otras investigaciones que realicen análisis detallados del hábitat y que determinen la disponibilidad de presas y la abundancia de los felinos en la región tumbesina.

\section{AGRADECIMIENTOS}

Este trabajo es un producto del proyecto "Evaluación del estado de conservación de los vertebrados terrestres mayores de las áreas protegidas de Manabí y Esmeraldas" financiado por la Universidad Laica Eloy Alfaro de Manabí. Agradecemos a cada una de las comunidades que se encuentran dentro del Parque Nacional Machalilla por su hospitalidad, a nuestro guía Ernesto Merchán por su apoyo y esfuerzo y a su familia por el aporte brindado en el trabajo.

\section{REFERENCIAS}

Briones-Salas M, Lira-Torres I, Carrera-Treviño R, Sánchez-Rojas G. 2016. Relative abundance and activity patterns of wild felids in Chimalapas rainforest. Oaxaca, Mexico. Therya. 7, 123-134.

Carrera-Treviño R, Astudillo-Sánchez Cl, Garza-Torres H, Martínez-García L, Soria-Díaz L. 2018. Interacciones temporales y espaciales de mesocarnivoros simpátricos en una reserva de biosfera: ¿convivencia o competencia?. Revista de Biología Tropical. 66, 996-1008. https://dx.doi.org/10.15517/ rbt.v66i3.30418

Ceballos G, Garcia A, Ehrlich PR. 2010. The sixth extinction crisis: Loss of animal populations and species. Journal of Cosmology, pp. 1821-1831.

Cervera L, Lizcano DJ, Parés V, Espinoza S, Poaquiza D, De la Montaña E, Griffith D. 2016. A camera trap assessment of terrestrial mammals in Machalilla National Park, western Ecuador. Check List. 12, 1868. http:// dx.doi.org/10.15560/12.2.1868

Clavijo A, Ramírez F. 2009. Taxonomía, distribución y estado de conservación de los felinos suramericanos: revisión monográfica. Boletín Científico Centro de Museos. Museo de Historia Natural, pp. 43-60. 
Di Bitetti M, De Angelo C, Di Blanco Y, Paviolo A. 2010. Niche partitioning and species coexistence in a Neotropical felid assemblage. Acta Ocologica. 34, 403-412. https:// doi:10.1016/j.actao.2010.04.001

Kiltie RA. 1984. Size ratios among sympatric neotropical cats. Oecologia. 61, 411416. https://doi.org/10.1007/bf00379644

Kolowski JM, Alonso A. 2010. Density and activity patterns of ocelots (Leopardus pardalis) in northern Peru and the impact of oil exploration activities. Biological Conservation. 143, 917-925. https://doi.org/10.1016/j.biocon.2009.12.039

MAE. 2017. Deforestación del Ecuador continental periodo 2014-2016. Quito, Ecuador: Ministerio del Ambiente.

Ortega-Andrade HM, Prieto-Torres DA, Gómez-Lora I, Lizcano DJ. 2015. Ecological and Geographical Analysis of the Distribution of the Mountain Tapir (Tapirus pinchaque) in Ecuador: Importance of Protected Areas in Future Scenarios of Global Warming. PLoS ONE 10(3): e0121137. https://doi.org10.1371/journal.pone.0121137

Payán-Garrido E, Soto-Vargas C. 2012. Los Felinos de Colombia. Ministerio de Ambiente y Desarrollo Sostenible, Instituto de Investigaciones de Recursos Biológicos Alexander von Humboldt y Panthera Colombia. pp. 48.

Pérez-Irineo G, Santos-Moreno A. 2014. Abundancia y patrones de actividad de felinos medianos (Felidae, Carnivora) en el sureste de México. The Southwestern Naturalist. 61, 33-39.

TEAM Network. 2011. Terrestrial Vertebrate Protocol Implementation Manual, v. 3.1. Arlington: Tropical Ecology, Assessment and Monitoring Network, Center for Applied Biodiversity Science, Conservation International.

Tirira DG, Brito J, Burneo SF, Moreno P, Pinto CM. 2019. Mamíferos del Ecuador: lista actualizada de especies / Mammals of Ecuador: Updated checklist species. Versión 2019.1. Asociación Ecuatoriana de Mastozoología. Quito, Ecuador. http://aem.mamiferosdelecuador.com (actualización / updated: 2019-09-30).

Editor: Jose F. Gonzalez-Maya Recibido 2020-05-05

Revisado 2020-06-05

Aceptado 2020-06-07

Publicado 2020-06-13 